\title{
DISEÑO DE UNA TÉCNICA PRESUPUESTAL PARA LAS MICROEMPRESAS AGROINDUSTRIALES DE ALIMENTOS DE LA CIUDAD DE SOGAMOSO, FUNDAMENTADO EN LA CARACTERIZACIÓN DE CADA UNA DE ELLAS \\ (Design of a budgetary technique for agroindustrial microenterprises of food, located in Sogamoso, based on the characterization of each one of them)
}

\author{
Josué Gustavo Rojas Sáenz, Jenny Mairena Herrera Rodríguez, Jorge Alí Oliveros Rodríguez
}

Escuela de Administración de Empresas, Grupo de Investigación Management, Semillero Seges.

(Recibido19 de Noviembre de 2012 y aceptado 13 de Marzo de 2013)

\begin{abstract}
Resumen:
Todas las actividades del hombre, desde su creación hasta nuestros días, han girado en torno al problema económico manifestado en una lucha continua por su subsistencia y desarrollo. Esta situación lo ha colocado como agente que produce o consume, por medio de decisiones de carácter individual que, obviamente, generan procesos que afectan a toda la sociedad. De ahí que las microempresas como factor de desarrollo social, determinen la forma como las personas y la sociedad buscan satisfacer de manera eficiente tanto las necesidades individuales como las colectivas. Es importante solucionar un número creciente de necesidades mediante el empleo de unos recursos productivos, escasos o limitados, los cuales tienen diferentes usos. En este proceso, se involucran decisiones de carácter técnico que determinan en un momento dado, las clases de bienes que deben producirse y distribuirse para la satisfacción de necesidades personales, grupales o, en general, de la sociedad como un todo. De lo anterior se deduce que una microempresa agroindustrial que no tenga diseñado un sistema presupuestal, estará en desventaja ante aquellas que sí lo hayan establecido. Efectivamente, el presupuesto permite cuantificar en términos monetarios y de forma anticipada, el resultado de decisiones y de diferentes objetivos trazados, de manera que pueda visualizarse su efecto en la organización para servir como herramienta que facilita el control administrativo, a fin de lograr mayor eficacia y eficiencia en las operaciones.
\end{abstract}

Paiaioras ciave: técnica presupuestal, presupuesto, planeación, operatividad.

\begin{abstract}
:
The activities of man, from his creation to the present day, have revolved around the economic problem manifested in a continuous struggle for his survival and development. This situation has placed him as an agent that produces or consumes, through individual decisions that, obviously, generate processes that affect the whole society. Hence, microenterprises as a factor of social development, determine the way as people and society seek to meet efficiently both the individual and collective needs It is important to solve a growing number of needs through the use of productive, scarce or limited resources, which have different uses. In this process, technical decisions are involved, which determine at any given time, the classes of goods to be produced and distributed to satisfy personal, group or social needs. It follows from the above that an agroindustrial microenterprise that has not designed a budget system, will be at a disadvantage to those that have established it. Indeed, the budget allows quantifying in monetary terms and in advance, the result of decisions and of different goals, so that it effect on the organization can be visualized to serve as a tool to facilitate management control, in order to achieve greater effectiveness and efficiency in operations.
\end{abstract}

Keywords: butgetary technique, butget, plamming, operability.

\section{DESCRIPCIÓN DEL PROYECTO}

El problema central que nos ocupa en esta investigación, es: ¿qué técnica presupuestal debe diseñarse para las microempresas agroindustriales de alimentos de la ciudad de Sogamoso, que les permita tener una herramienta moderna de planeación y control? Esta investigación se justifica por la importancia que posee la técnica presupuestal en la planeación, la previsión y el control, como factores fundamentales de la gestión de las empresas y, por ende, de las microempresas agroindustriales.
El diseño de una técnica presupuestal acorde con las necesidades de la microempresa agroindustrial, no es simplemente una cuestión numérica, también es un asunto que se refiere a la determinación de los parámetros dentro de los cuales se puede lograr el éxito o el fracaso, cuando se comparan los resultados obtenidos en la operatividad de la microempresa. Parámetros que deben establecerse al incorporar una adecuada técnica presupuestal, mediante la cual se analicen las diferentes desviaciones entre los objetivos trazados y los resultados obtenidos.
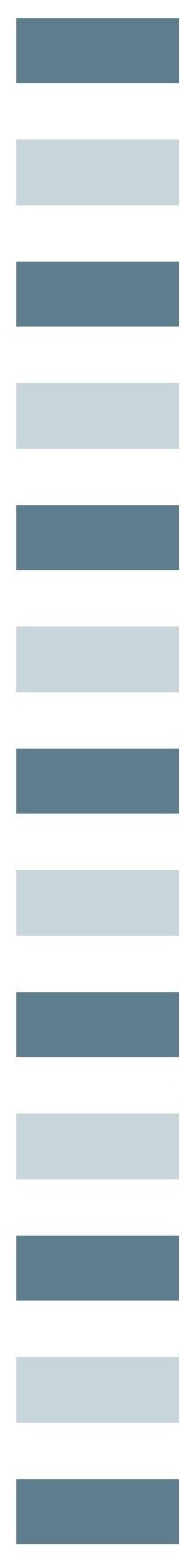

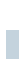


Además, para el desarrollo del proyecto se toma como premisa importante el proceso de planificación de la microempresa agroindustrial; punto que resulta ser uno de los más críticos, puesto que no puede pensarse en un buen funcionamiento de esta, sin un mínimo proceso de planificación de las actividades.

En la planeación se puede distinguir lo que es el proceso y lo que es el contenido. El proceso consiste en establecer los escenarios y fijar los objetivos, mientras que el contenido está formado por el plan de mercadeo, el plan de insumos y el plan financiero. Por lo tanto, la microempresa agroindustrial debe ejecutar ambas partes, ya que esta debe conocer la competencia, sus clientes, sus proveedores, como también debe enterarse de los posibles efectos que generarían la fijación y el manejo de los precios. Con base en lo anterior, cabe resaltar que dentro de la actividad de planificar, es importante la variable tiempo, más específicamente, el periodo de tiempo.

A priori, parece más sencillo acotar el futuro "próximo" que el futuro "lejano". Se considera que de este último, se ocupa la planificación a largo plazo o planificación estratégica, mientras que el futuro "próximo" o corto plazo debe relacionarse con la planificación presupuestaria. Y es precisamente aquí de donde parte la presente investigación, ya que el presupuesto debe definir los objetivos y metas a corto plazo (futuro próximo) que la microempresa agroindustrial ha establecido, y los medios para lograrlos.

\section{METODOLOGÍA}

En este contexto se describe la manera como se adelanta el desarrollo de la investigación. La planeación tuvo el propósito de cumplir con los objetivos fijados. La investigación conlleva la consulta sobre las microempresas agroindustriales en lo referente a su ubicación, tamaño, recursos y demás variables que sean relevantes, pero haciendo énfasis en el evento de si las microempresas agroindustriales de alimentos emplean o no algún tipo de técnica presupuestal, ya que la investigación apunta al "diseño de una técnica presupuestal para las microempresas agroindustriales de alimentos de la ciudad de Sogamoso, fundamentada en la caracterización de cada una de ellas".

Se empleó el modelo investigativo de naturaleza exploratoriodescriptivo, partiendo del criterio que el resultado es de tipo teórico-práctico. En este estudio se siguieron las siguientes etapas:

1. Observación: mediante esta técnica se obtuvo la información necesaria que permitió determinar la rama productiva o actividades específicas de las microempresas agroindustriales de alimentos.

2. Método analítico: este método facilitó la clasificación de la información recolectada, convirtiéndose en una base real para entender de forma más simple los patrones descubiertos en la investigación.

3. Método deductivo: después de identificar los detalles relevantes en la investigación, se procedió a enlazarlos con los objetivos propuestos, para poder determinar así, si la investigación realizada correspondió y concluyó de acuerdo con la que se planteó inicialmente, y, por ende, mostrar en forma práctica los resultados obtenidos.

4. Método descriptivo: con esta técnica se pretendió conseguir la información necesaria, que constituye la base del análisis de presupuesto. La cual se editará, codificará, tabulará, depurará y almacenará para el correspondiente análisis de la información y sus resultados.

\subsection{Fuentes de información}

La información necesaria para la presente investigación proviene de fuentes de información primaria y secundaria.

Fuentes de información primaria: la información primaria se requirió por cuanto el estudio es de tipo teórico-práctico, lo que implica el uso de técnicas adecuadas y procedimientos claros, para obtener la información adecuada. Las fuentes primarias estuvieron conformadas por gerentes, empresarios, propietarios o la persona encargada del manejo de la microempresa agroindustrial de alimentos.

Fuentes de información secundaria: se recurrió a resúmenes, revistas, libros, documentos, informes presentados por los diferentes gremios que tienen injerencia en el manejo de los sectores primario y secundario de la economía, así como en el desarrollo agroindustrial de Sogamoso. Se revisaron las diferentes publicaciones que se encuentran en bibliotecas, en entidades gubernamentales y no gubernamentales, en archivos, etc., y que ofrecen información con respecto a la temática planteada en el proyecto de investigación. La información también se obtuvo por medio de la Cámara de Comercio de esta ciudad.

Para la investigación se tuvo en cuenta, de forma más específica, la literatura relacionada con: presupuesto y control de las empresas, gerencia y administración financiera, contabilidad administrativa, procesos administrativos y demás temas sobre los cuales se centra la investigación.

\subsection{Instrumento de recolección de información}

Se utilizó un formulario diseñado para adelantar la entrevista personal y, desde luego, se acudió a la observación directa.

Este instrumento fue aplicado a diez microempresas agroindustriales de alimentos, por intermedio de los gerentes y representantes legales, que corresponde a la muestra tomada para tal fin.

El instrumento aplicado se diseñó con base en tres criterios: la información general, las variables incorporadas alrededor del presente trabajo y los tópicos específicos que orientaron las respuestas del encuestado.

\subsection{Población y muestra}

Sobre el fundamento de contar con una población fácilmente cuantificable, es decir, de una población finita $(\mathrm{N}=10$, según la información recopilada de la Cámara de Comercio), no fue necesario el cálculo de una muestra para hacer la inferencia acerca de la población, lo cual significó que se manejó un mayor grado de certeza. En concreto, se consideró la población o universo a las microempresas Agroindustriales ubicadas en la ciudad de Sogamoso. La unidad informante estuvo constituida por gerentes, propietarios o por la persona encargada del manejo de la unidad productiva. 


\subsection{Metodología y procedimiento de investigación}

Una vez terminada la necesidad de información, planteado los objetivos y descrito el problema en estudio, se procedió al desarrollo de las siguientes actividades:

- Recolección de la información.

- Sistematización y procesamiento de la información.

- Análisis de los resultados obtenidos.

Recolección de información: se estableció un tipo de investigación descriptiva o concluyente, dado que esta permitirá crear un vínculo entre los objetivos planteados y la información que se obtuvo con la realización de la encuesta.

Identificadas las unidades de investigación, se procedió a la recolección de la información, mediante la entrevista personal y la observación directa.

Una vez recolectados los datos, se organizaron, editaron y codificaron, para finalmente obtener la información base para el análisis de resultados.

\section{REFERENTE TEÓRICO}

Según Jorge Burbano (1990), el presupuesto es la estimación programada de manera sistemática, de las condiciones de operación y de los resultados que se pretenden obtener por un organismo en un periodo determinado. También dice que el presupuesto es una expresión cuantitativa formal de los objetivos que se propone alcanzar la administración de la empresa en un periodo, con la adopción de las estrategias necesarias para lograrlos. Pocas veces un presupuesto es algo aislado, más bien es un resultado del proceso gerencial que consiste en establecer objetivos y estrategias y en elaborar planes (Sweeny, 1984). Especialmente se encuentra íntimamente relacionado con la planeación financiera. Por lo tanto, el presupuesto puede considerarse una parte importante del clásico ciclo administrativo de planear, actuar y controlar o, más específicamente, como parte de un sistema total de administración que incluye:

- Formulación y puesta en práctica de estrategias.

- Sistemas de planeación.

- Sistemas presupuestales.

- Organización.

- Sistemas de producción y mercadotecnia.

- Sistemas de información y control.

Los presupuestos se basan en la convicción de que una administración puede y debe controlar el futuro de la empresa mediante la toma de una serie continua de decisiones bien concebidas:

Prosperidad planeada versus caso fortuito: el presupuesto es una herramienta para largo plazo. Muestra las actividades de la empresa en forma general y está encausada hacia unos objetivos generales. La planeación supone que el estado futuro de una entidad podrá ser mejorado por una acción administrativa continua. Con base en esto, Rusell L. Ackoff en su libro A Concept of Corporate Planning (1970), sugiere que durante el proceso de planeación, la administración de una entidad debe emplear tres tipos diferentes de proyecciones, a saber:

a. - Proyección de referencia. ¿Cuál sería el estado futuro de una entidad, de una empresa, si no se hace nada nuevo, es decir, si no se planifica?

b. - Proyección deseada. Es una especificación de los "sueños", de las "esperanzas", con relación al estado futuro de la entidad, es decir, las aspiraciones de la empresa.

c. - Proyección planeada. Es el análisis de las posibilidades que tiene la entidad para lograr la proyección deseada en forma realista, (Del Río, 2000).

\section{¿Por qué debe caracterizarse cada una de las microempresas?}

Porque tienen necesidades distintas, así como elementos que las componen de forma diferente; no hay dos empresas similares o iguales en su infraestructura. La forma, contenido y profundidad del presupuesto siempre van ligados con los objetivos, necesidades y organización de la empresa. Su preparación se basa en el principio de que todas las transacciones de la entidad están íntimamente relacionadas entre sí.

Así pues, los presupuestos son base para las ventas, ya que "Un pronóstico se convierte en un plan de ventas cuando la administración incorpora en él juicios, estrategias planeadas, compromiso de recursos y la dedicación administrativa a las acciones agresivas para lograr las metas de ventas" (Welsch, s. f.), de modo que los presupuestos significan más que ver hacia el futuro, indican que se tiene la certeza de lo que realmente se espera, con el mínimo riesgo.

\section{CONCLUSIONES}

Como el estudio pretendió determinar si las mencionadas empresas efectúan una planeación de sus actividades operacionales, hay que anotar que esta no se ve reflejada en los resultados de la correspondiente investigación.

Debe tenerse en cuenta que la operatividad de la empresa, en la mayoría de ellas, se lleva a cabo con base en la experiencia adquirida durante los años del propietario de la misma, mas no en criterios técnicos en lo administrativo, económico y de comercialización.

La mayoría de las empresas encuestadas no aplican ninguna técnica presupuestal, por desconocimiento de como se formula, se desarrolla y se evalúa un presupuesto general.

El empresario es reacio a brindar una información concreta y veraz, debido a la desconfianza que siente hacia los encuestadores.
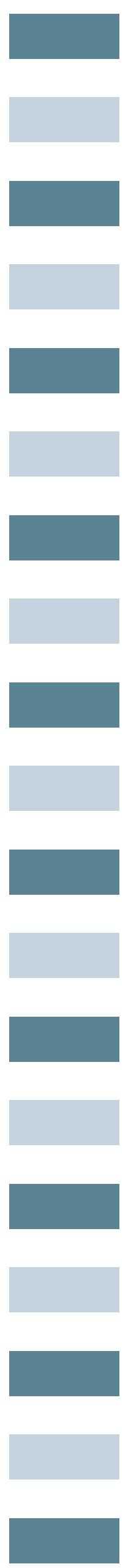


\section{RECOMENDACIONES}

Se sugiere establecer unos principios claros y concisos mediante los cuales pueda realmente hacerse una planeación bajo criterios que permitan el buen rendimiento de la empresa. Es necesario que los propietarios de la mayoría de las empresas, establezcan unos criterios claros y técnicos sobre el desarrollo de procesos administrativos mediante los cuales se pueda ver un mayor desenvolvimiento en la ejecución y valoración de la empresa.

La mayoría de las empresas encuestadas no aplican ninguna técnica presupuestal de tipo operacional o económico, ni un presupuesto dentro de los criterios financieros.

Aquellas empresas que facilitan la información, pueden ser realimentadas con los resultados obtenidos mediante la investigación efectuada. Se recomienda asesorar a los empresarios en la formulación, elaboración y aplicación de alguna de las técnicas presupuestales, por intermedio de la Escuela de Administración de Empresas de la Uptc, Seccional Sogamoso.

\section{REFERENCIAS}

Ackoff, R. (1970). A concept of corporate planning. New York: John Wiley \& Sons, Wiley-Interscience.

Burbano, J. (1990). Presupuestos. Bogotá: Mac Graw Hill.

Del Río, G. (2000). El Presupuesto. México: ECAFSA.

Cámara de Comercio de Sogamoso. (2012). Base de datos.

Dromi, J. (1988). Presupuesto y costo de inversión. Buenos Aires: Astrea.

Pereda, M. (1980). Presupuesto base cero. Madrid: Cirde.

Rojas, S. J. (2007). Instrumento metodológico para la elaboración, ejecución y control de presupuesto maestro de pymes agroindustriales. Trabajo de Grado de Maestría. Universidad Nacional de Colombia, Bogotá.

Sweeny, R. R. (1984). Manual de presupuestos. México: McGraw-Hill.

Welsch, G. (s.f.). Presupuestos: planificación y control de utilidades. Madrid: Dossat, Prentice Hall Internacional Inc. 